\title{
Suzaku observations of early-type galaxies
}

\author{
K. Matsushita ${ }^{1}$, Y. Fukazawa ${ }^{2}$, K. Hayashi ${ }^{2}$, S. Konami ${ }^{1}$, \\ R. Nagino ${ }^{1}$, T. Ohashi ${ }^{3}$, Y. Tawara ${ }^{4}$ and M. Tozuka ${ }^{1}$
}

${ }^{1}$ Department of Physics, Tokyo University of Science, 1-3 Kagurazaka, Shinjyuku-ku, Tokyo 162-8601, Japan, email: matusita@rs.kagu.tus.ac.jp

${ }^{2}$ Department of Physical Science, Hiroshima University, 1-3-1 Kagamiyama, Higashi-Hiroshima, Hiroshima 739-8526, Japan

${ }^{3}$ Department of Physics, Tokyo Metropolitan University, 1-1 Minami-Osawa, Hachioji, Tokyo 192-0397, Japan

${ }^{4}$ Department of Physics, Nagoya University, Nagoya, Japan

The metal abundances in the hot X-ray emitting interstellar medium (ISM) of early-type galaxies give us important information about the present metal supply into the ISM through supernovae ( $\mathrm{SNe}$ ) Ia and stellar mass loss. In addition, $\mathrm{O}$ and $\mathrm{Mg}$ abundances should reflect the stellar metallicity and enable us to directly look into the formation history of these galaxies. The XIS instrument onboard the Suzaku satellite has an improved line spread function due to a very small low-pulse-height tail below $1 \mathrm{keV}$ coupled with a very low background.

We derived abundance pattern of $\mathrm{O}, \mathrm{Ne}, \mathrm{Mg}$ and Fe of ISM of four elliptical galaxies, NGC 720 (Tawara et al. 2007), NGC 1399 (Matsushita et al. 2007a), NGC 1404 (Matsushita et al. 2007a), NGC 4636 (Hayashi et al. 2009), and two S0 galaxies, NGC 1316 (Konami et al. 2009), and NGC 4382 (Nagino et al. 2009) observed with Suzaku, and compared with those of two cD galaxies, M 87 (Matsushita et al. 2003), and NGC 4696 (Matsushita et al. 2007b) observed with XMM.

The Fe abundances of the ISM of these galaxies are about $0.5-1$ solar, indicating that the present $\mathrm{SN}$ Ia rate is low. The $\mathrm{O}, \mathrm{Ne}$ and $\mathrm{Mg}$ abundances are consistent with stellar metallicity. The abundance patterns of the ellipticals and cDs, and NGC 1316 are not so different from the solar pattern, using new solar abundance by Loddars (2003), and consistent with a mixture of $\mathrm{SNe}$ Ia and metal-poor Galactic stars. These galaxies are giant galaxies and their ISM temperatures are $0.6 \sim 1 \mathrm{keV}$. In contrast, a S0 galaxy, NGC 4382, with an ISM temperature of $0.3 \mathrm{keV}$, has a smaller $\mathrm{O} / \mathrm{Fe}$ ratio in the ISM. This result means that the ISM in this galaxy contains more SNIa products.

\section{References}

Hayashi, K., Fukazawa, Y., Tozuka, M., Nishino, S., Matsushita, K., Takei, Y., \& Arnaud, K. A. 2009, PASJ in press, arXiv:0907.2283

Konami, S., in preparation

Lodders, K. et al. 2003, ApJ 591, 1220

Matsushita, K., Finoguenov, A., \& Böhringer, H. 2003, A\&\&A 401, 443

Matsushita, K. et al. 2007a, PASJ 59, 327

Matsushita, K., Böhringer, H., Takahashi, I., \& Ikebe, Y. 2007b, A 8 A 462, 953

Nagino, R. \& Matsushita, K. in preparation

Tawara, Y., Matsumoto, C., Tozuka, M., Fukazawa, Y., Matsushita, K., \& Anabuki, N. 2008, PASJ 60, 307 